\title{
Conformational epitopes of myelin oligodendrocyte glycoprotein are targets of potentially pathogenic antibody responses in multiple sclerosis
}

Til Menge ${ }^{1,2^{*}}$, Patrice H Lalive ${ }^{1,3}, \mathrm{H}$-Christian von Büdingen ${ }^{1}$ and Claude P Genain ${ }^{1}$

\begin{abstract}
Background: Myelin/oligodendrocyte glycoprotein (MOG) is a putative autoantigen in multiple sclerosis (MS). Establishing the pathological relevance and validity of anti-MOG antibodies as biomarkers has yielded conflicting reports mainly due to different MOG isoforms used in different studies. Because epitope specificity may be a key factor determining anti-MOG reactivity we aimed at identifying a priori immunodominant MOG epitopes by monoclonal antibodies (mAbs) and at assessing clinical relevance of these epitopes in MS.

Methods: Sera of 325 MS patients, 69 patients with clinically isolated syndrome and 164 healthy controls were assayed by quantitative, high-throughput ELISA for reactivity to 3 different MOG isoforms, and quantitative titers correlated with clinical characteristics. mAbs defined unique immunodominant epitopes distinct to each of the isoforms.

Results: In the majority of human samples anti-MOG levels were skewed towards low titers. However, in $8.2 \%$ of samples high-titer anti-MOG antibodies were identified. In contrast to anti-MOG reactivity observed in a mouse model of MS, in patients with MS these never reacted with ubiquitously exposed epitopes. Moreover, in patients with relapsing-remitting MS high-titer anti-MOG IgG correlated with disability (EDSS; Spearman $r=0.574 ; p=0.025$ ).

Conclusions: Thus high-titer reactivity likely represents high-affinity antibodies against pathologically relevant MOG epitopes, that are only present in a small proportion of patients with MS. Our study provides valuable information about requirements of anti-MOG reactivity for being regarded as a prognostic biomarker in a subtype of MS.
\end{abstract}

Keywords: Antibodies, Autoimmunity, Multiple sclerosis, Myelin, Biomarkers

\section{Introduction}

Autoantibodies directed against myelin antigens have been a long-standing focus of interest in multiple sclerosis (MS) research, especially those binding to myelin oligodendrocyte glycoprotein (MOG). MOG is predominantly expressed in the CNS, and is exposed on the outermost lamellae of the myelin sheath thus readily available for a humoral immune attack [1]. MOG induces demyelinating experimental allergic encephalomyelitis (EAE), the animal model of MS, in a variety of

\footnotetext{
* Correspondence: menge@uni-duesseldorf.de

${ }^{1}$ Neuroimmunology Laboratories, Department of Neurology, University of California San Francisco, CA, USA

Full list of author information is available at the end of the article
}

species both by active immunization and by passively transferred anti-MOG antibodies (reviewed by $[1,2]$ ). Only those anti-MOG antibodies directed against conformational epitopes, as opposed to linear epitopes, appear to be pathogenic in EAE [3-5]. Recently, it was shown that the murine monoclonal antibody ( $\mathrm{mAb}$ ) 8.18.c5 specific for rat MOG, that confers demyelination, maps to a discontinuous epitope of the surface exposed FG loop of rat MOG, that is also exposed on murine and human MOG [6].

To date, the measurement of serum anti-MOG antibodies using various techniques and different MOG preparations has resulted in inconsistent results and limited reproducibility (reviewed by $[7,8]$ ). This study was thus 
designed to assess the MOG epitope usage in humans employing a novel quantitative high-throughput ELISA. The serum anti-MOG antibody responses of 325 patients with MS, 69 patients with a first demyelinating event (clinically isolated syndrome, CIS) and 164 healthy controls (HC) were assayed. Three isoforms of recombinant MOG were generated and the differential exposure of immunodominant epitopes characterized by a panel of monoclonal anti-MOG antibodies. Restricted patterns of anti-MOG reactivity could be observed in samples with sustained anti-MOG reactivity at high serum dilutions, defining high-titer reactivity. In this cohort we find that anti-MOG antibody levels strongly correlate with disease severity.

\section{Materials and methods Antigens and Antibodies}

Three recombinant human MOG isoforms were used for the study. The first, spanning the extracellular domain, amino acids $1-125$, $\left(\mathrm{rhMOG}_{125}\right)$ was expressed and purified under physiological conditions as described previously [9]. Secondly, a seven amino acids shorter rhMOG protein, spanning the amino acids 1-118 $\left(\right.$ rhMOG $\left._{118}\right)$ was created by usage of a different 3'-end primer: 5'-ATCCATGAGATCTAGGATCTTCTACTTT CAATTCCATTGCTGCC-3', and was expressed and purified as above. Finally, recombinant rat MOG, amino acids 1-125 (ratMOG 125 ) was produced in E. coli and purified as described previously [10]. Purity was confirmed to be $>95 \%$ by SDS-PAGE (additional file 1) and correct folding ascertained by circular dichroism (additional file 1) [11,12].

The murine monoclonal IgG 8.18C5 against native rat MOG was a gift of Dr. Chris Linington [13]. The marmoset Fab-fragments (Fabs) designated M26, M3-24, and M3-8 derived from a ratMOG 125 -immune animal were generated in our laboratory as described previously [10].

\section{Patients}

325 MS patients meeting the diagnostic criteria for clinically definite MS [14,15], and 69 patients with a first demyelinating were recruited for this study [15]. 36\% of the MS patients were treated with either interferon beta or glatiramer acetate at the time of sampling. Patients treated with glucocorticoids within three months or on immunosuppressive therapy within six months of phlebotomy were excluded. 164 volunteers served as healthy controls (HC). Informed consent was obtained from all subjects, and the study was conducted in accordance with Institutional Review Board approval. The clinical characteristics of the patients and $\mathrm{HC}$ are summarized in the table contained in additional file 2. Blood was drawn by venipuncture and clotted serum stored at $-40^{\circ} \mathrm{C}$.

\section{High-throughput ELISA}

Optimal protein concentrations for coating were determined in preliminary experiments; $0.5 \mu \mathrm{g}$ of $\mathrm{rhMOG}_{125}$ and $\mathrm{rhMOG}_{118}$ and $1.0 \mu \mathrm{g}$ of ratMOG $\mathrm{MO}_{125}$ were coated in PBS overnight on a single 384-well microtiter assay plate (Maxisorb, Nunc, Rochester, NY). Control wells were coated with $1.0 \mu \mathrm{g}$ BSA. To quantify the antibody reactivity, an IgG standard curve was created by coating human IgG (I4506, Sigma, St. Louis, MO) in two-fold dilutions. After washing, plates were blocked for 2 hours with 1\% BSA in PBS supplemented with $0.05 \%$ Tween 20. Then, human sera were added at three dilutions starting at $1 / 200$, and incubated for $90 \mathrm{~min}$. A positive control known to be reactive to all three rMOG isoforms, and a negative control omitting serum were included. Bound antibodies were detected by an alkaline phosphatase-labeled anti-human IgG (A9544, Sigma) and the optical density (OD) read at $405 \mathrm{~nm}$ wavelength in a microplate reader (SpectraMax, Molecular Devices, Sunnyvale, CA) after 30 minute incubation with paranitrophenyl phosphate (Moss, Pasadena, MD). All samples were tested coded in a blinded fashion in duplicates, standard curves in quadruplicates; incubation was at room temperature (RT), except coating which was at $4^{\circ} \mathrm{C}$. Sample handling and ELISA procedures were performed by a robotic workstation (Biomek FX, Beckman Coulter, Fullerton, CA). MOG-ELISA for the monoclonal reagents (8.18c5 and Fab fragments) were performed in 96-well Maxisorb plates according to the protocol as outlined above. 8.18c5 was detected by an anti-mouse IgG (A9044, Sigma) and the Fabs by Protein L (Pierce). Both were peroxidase labeled and developed by 3,3',5,5'tetramethylbenzidine (Pierce) and the OD read at 450 $\mathrm{nm}$ wavelength.

\section{Denaturing anti-MOG ELISA}

To further demonstrate, that conformational MOG-epitopes are conserved in regular ELISA assays as described above, we compared binding of anti-MOG antibodies to recombinant MOG antigens coated under native (previous section) and denaturing conditions. The rMOG isoforms were coated as outlined above in 96-well plates. After washing coated MOG was denatured by incubation with $8 \mathrm{M}$ urea supplemented with $10 \mathrm{mM}$ dithiothreitol (DTT) for 4 hours at RT followed by addition of $25 \mathrm{mM}$ 2-iodoacetamide (IDAA) for fixation. After overnight incubation at RT and extensive washing wells were blocked as above, subjected to either 8.18c5 or anti-His mAb (Invitrogen, Carlsbad, CA), and binding was detected as above.

\section{Data processing and statistical analysis}

The ODs were corrected for the individual background binding and the amount of specific IgG bound to the 
well interpolated from the on-plate standard curve. Additionally, results were expressed as the signal-tobackground binding ratio (BR), calculated as the ratio of OD signal/background where applicable, as were results for $8.18 \mathrm{c} 5$ and the Fabs.

For the assessment of high-titer reactivity, samples above to $95^{\text {th }}$ percentile of IgG concentrations were tested in a separate experiment in serial dilutions, those with $B R s \geq 2$ at 1/3,200 dilution (i.e. signals against MOG that were at least two-fold above the BSA signal) were identified and their mean BR at 1/ 800 dilution defined as the cut-off for high-titer reactivity. This procedure was done for all three MOG isoforms. This procedure is one of the rigorous methods accepted for defining subtype cut-offs with stringent criteria.

Statistical analysis was conducted using Prism 4.0 (GraphPad, San Diego, CA). Categorical variables were compared using the $\chi^{2}$-test, continuous variables using ANOVA and ordinal as well as not normally distributed continuous variables by Kruskal-Wallis testing. The Student-Newman-Keuls method and Dunn's test were used to determine differences in between groups.

\section{Results}

Demonstration of conformationally coated MOG in ELISA Prior to assessing the putative immunodominant MOG epitopes it was shown that coating of MOG to the ELISA plate by electrostatic forces did not denature the protein. Making use of the mAb 8.18c5 that is known to exclusively bind to conformational, but not linear MOG epitopes [5,6], recombinant MOG was denatured by urea and DTT after coating, and the linearized polypeptide fixated by the alkylating sulfhydryl reagent IDAA that binds covalently with cysteine and thus prevents renaturing of MOG. While $8.18 \mathrm{c} 5$ reactivity is lost after denaturing/fixating, anti-His $\mathrm{mAb}$ reactivity is sustained indicating the MOG polypeptide remains coated in the wells (exemplified for rhMOG 118 ; Figure 1).

Thus, coating does not denature MOG to an extent that pathogenic conformational epitopes are destroyed or become unexposed. This however, does not prevent antibodies reactive to linear epitopes to bind to coated MOG as exemplified for human sample \#1139 (Figure 1 ) and as shown previously [3,9]. Because at least half of the reactivity of serum \#1139 is lost after denaturing of rhMOG $_{118}$ the samples contains both antibodies against linear epitopes and those exclusively reactive to conformational epitopes (Figure 1).

\section{Definition of distinct epitopes exposed on the different MOG isoforms}

Despite $90 \%$ sequence homology between rhMOG $_{125}$ and ratMOG ${ }_{125}$, and a mere 7 amino acid difference in

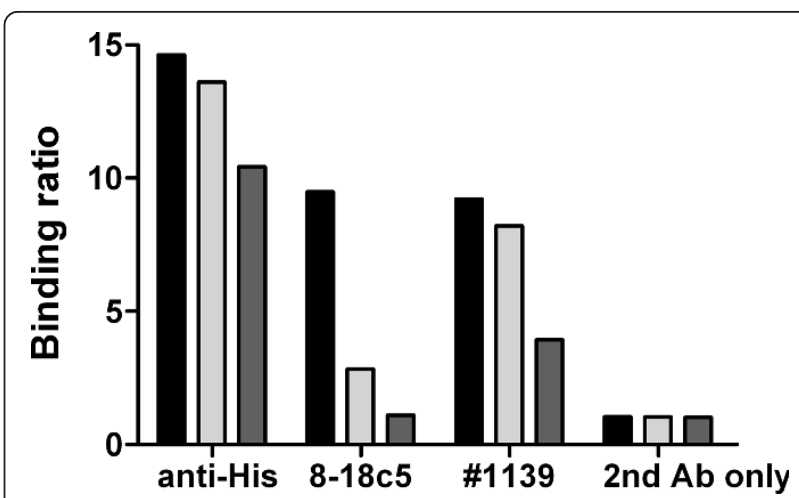

Figure 1 Denaturing ELISA. Comparison of rhMOG 118 coated under physiological conditions in PBS (black bars), after partial denaturing of rhMOG 118 by incubation with $8 \mathrm{M}$ urea (light grey bars) and after irreversibly completely denaturing by incubation with $8 \mathrm{M}$ urea, $10 \mathrm{mM}$ DTT, 25 mM IDAA (dark grey bars). Results are expressed as binding ratios as described in the methods section. Anti-His denotes a mAb specific for 6-mer histidine peptide tag; $\# 1139$ is a human serum serving as positive control in the quantitative ELISA.

length between $\mathrm{rhMOG}_{118}$ and $\mathrm{rhMOG}_{125}$, each of the antigenic isoforms used in this ELISA displayed unique immunodominant epitopes of MOG or combinations thereof, as demonstrated by the mAb $8.18 \mathrm{c} 5$ and the monoclonal Fabs M26, M3-24 and M3-8:

- 8.18c5 and M26 bound equally well to all isoforms, defining epitopes commonly exposed on all three MOG isoforms (Figure $2 \mathrm{~A}+\mathrm{B}$ ).

- A second epitope, defined by the marmoset Fab M3-24, is unique to $\mathrm{rhMOG}_{125}$ and ratMOG 125 , and is not exposed on $\mathrm{rhMOG}_{118}$; hence this epitope is species-independent, but dependent on the length of the protein (Figure $2 \mathrm{C}$ ).

- Thirdly, the Fab M3-8 recognizes an epitope uniquely exposed on ratMOG ${ }_{125}$, but on neither of the human proteins; hence this epitope is speciesdependent, (Figure 2D).

It has been previously shown that these Fabs do not inhibit each other's binding or binding of $8.18 \mathrm{c} 5$, nor do they recognize linear MOG-derived peptides, which corroborates our interpretation that the different MOG isoforms expose distinct, conformation dependent, immunodominant epitopes [10]. Thus, the use of these three different recombinant isoforms affords to measure antibodies against specific conformational epitopes of MOG in serum.

\section{Anti-MOG reactivity in healthy controls and cohorts of patients with MS}

The quantitative results for antibodies against the 3 different MOG isoforms in 325 patients with MS, 69 CIS 

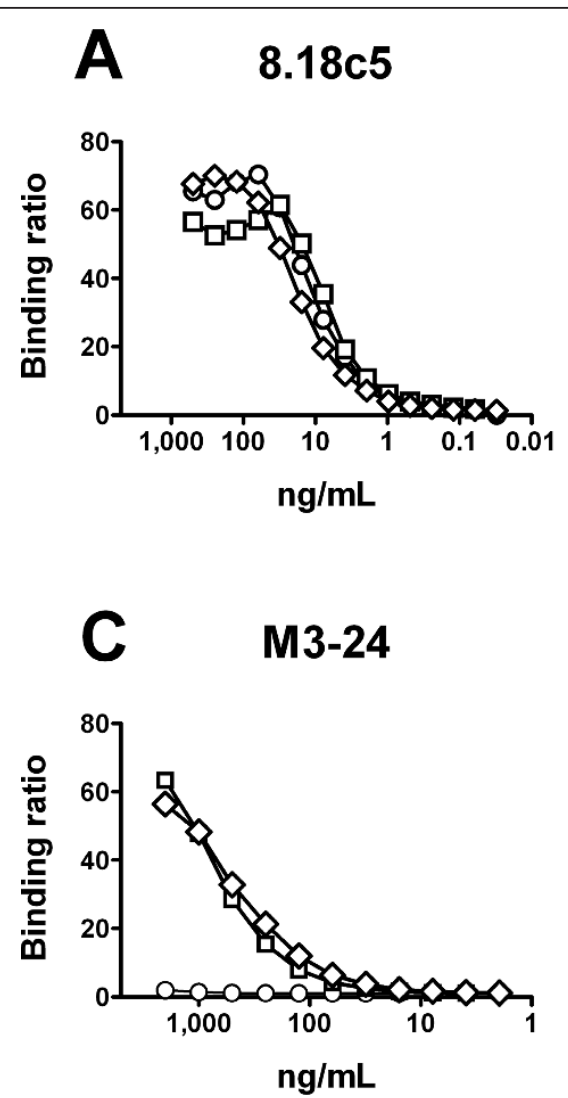
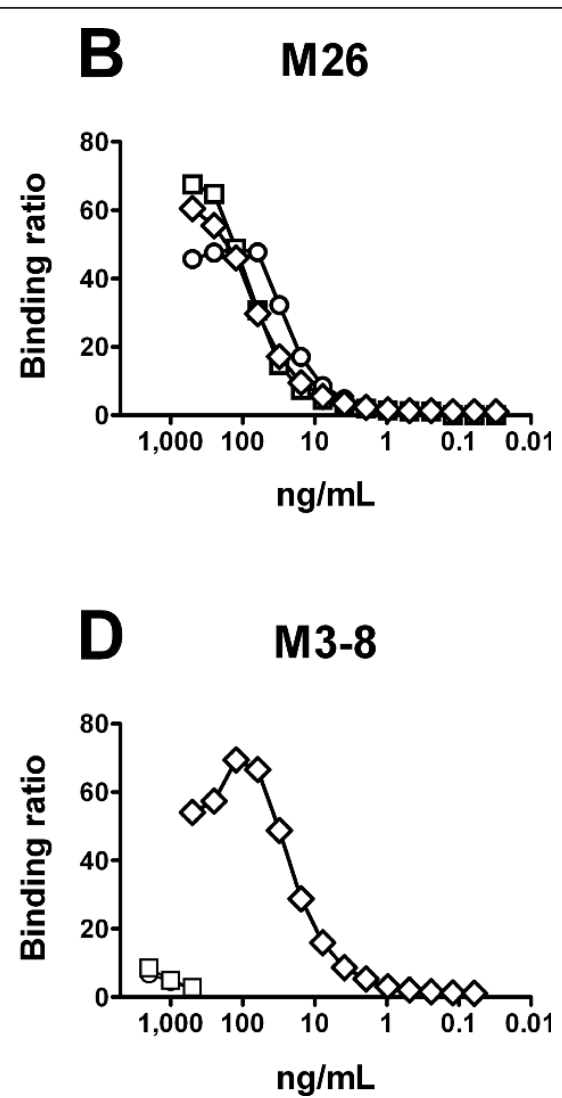

Figure 2 Monoclonal reagents define distinct epitopes on MOG. Serial two-fold dilution series of the mouse monoclonal antibody $8.18 c 5$ $(\mathrm{A})$, and the marmoset-derived Fab fragments M26 (B), M3-24 (C) and M3-8 (D) against rhMOG 118 (-O-), rhMOG 125 (- $\square-)$ and ratMOG $125(-\Delta-)$.

patients and 164 HC samples obtained by high-throughput ELISA are summarized in Figure 3. Anti-MOG IgG concentrations below $5.0 \mu \mathrm{g} / \mathrm{mL}$ were over-represented, resulting in a skewed distribution $\left(75^{\text {th }}\right.$ percentile $<5.1$ $\mu \mathrm{g} / \mathrm{mL}$ for all groups; Figure 3). Anti-rhMOG ${ }_{125}$ reactivity was significantly elevated in the CIS patient group compared to MS and HC ( $\mathrm{p}<0.05)$, and anti-rat$\mathrm{MOG}_{125}$ significantly higher in MS compared to HC (p $<0.05$ ); however, extensive overlap between groups was observed and the clinical relevance of this finding remains unclear (Figure 3).

Importantly, age, gender, disease duration, or treatment could be excluded as confounding factors in any of the MS subgroups or HC (data not shown).

\section{Identification and fine epitope specificity of subjects with} high-titer anti-MOG IgG

Because of the skewed distribution in favor of low serum concentration antibodies, we sought to identify high-titer, higher affinity samples reasoning that these would be immunologically relevant antibodies reflecting a true immune response, and investigated their reactivity patterns. The quantitative assay with multiple serial dilutions is capable of providing this information in two ways; first, it allows to measure actual antibody concentrations; second, it allows differentiating antibodies that retain reactivity at high dilutions, which may be indicative of higher affinity and could not otherwise be measured in a complex mixture of serum IgG. To specifically identify samples with high-titer reactivity and to differentiate those from the ones that show reactivity only at lower dilutions, we defined cut-offs for BR for the 1/800 dilution, derived from samples with antiMOG IgG concentrations above the $95^{\text {th }}$ percentile and with sustained antibody reactivity at 1/3,200 dilution (see Methods; additional file 3). Applying these cut-offs, $8.2 \%$ of all $\mathrm{HC}$, CIS and MS samples were identified as high-titer reactive; differences between groups were not statistically significant (table 1).

Detailed analysis of the high-titer sera revealed that the majority of these reacted with only one of the human MOG isoforms, either rhMOG $_{118}$ or rhMOG $_{125}$ ("monospecific"), or with both of them ("oligospecific", table 1). Concomitant reactivity to all three MOG antigens was only found in one HC sample (table 1). This pattern was consistent in all groups, 


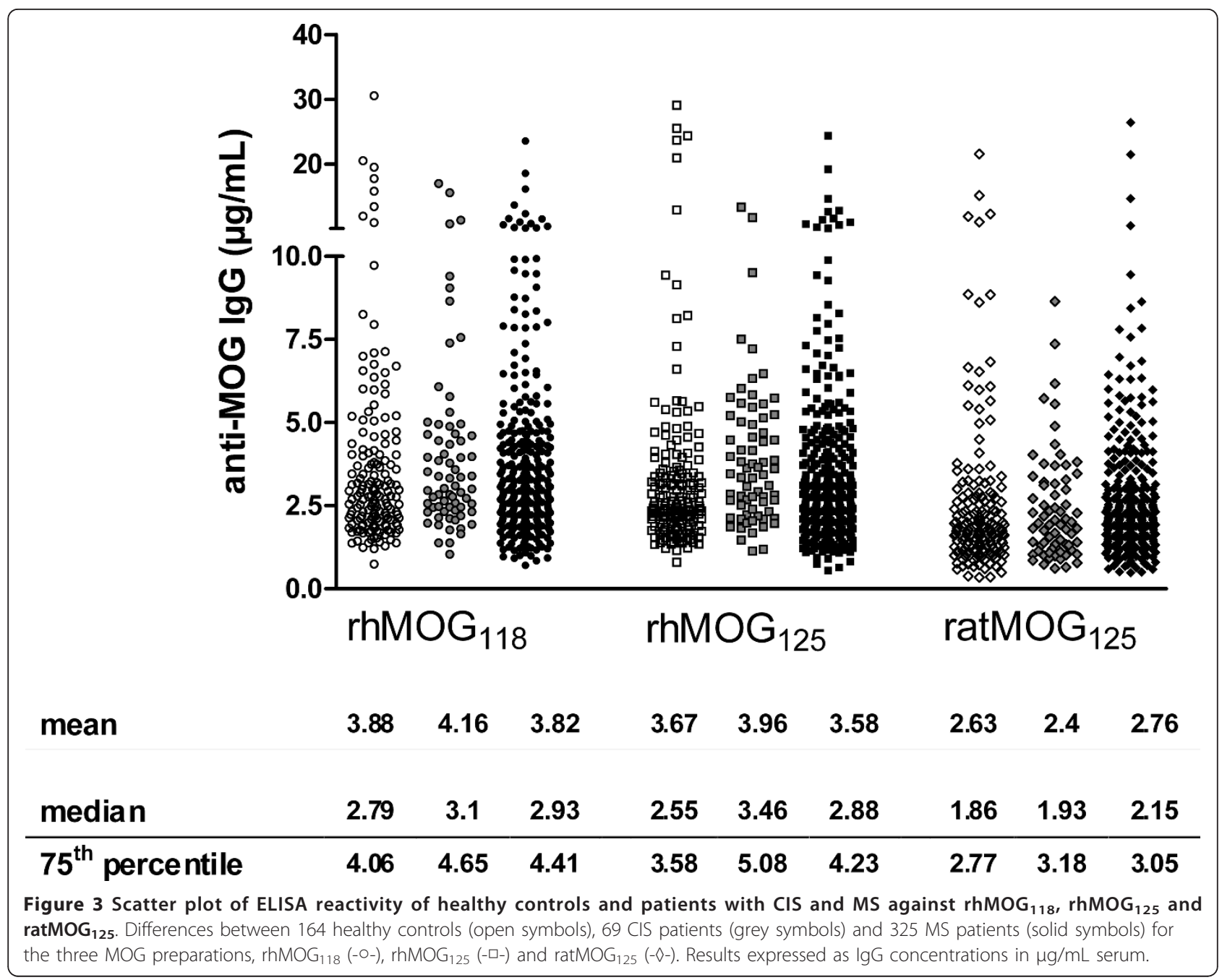

and there were no differences between early disease (CIS) and chronic forms of MS (SPMS, PPMS). This dichotomy of reactivity is corroborated by the observation that anti-rhMOG 118 IgG concentrations correlated well with the respective anti-rhMOG 125 IgG concentrations in the oligospecific high-titer samples (Spearman $\mathrm{r}=0.574 ; \mathrm{p}=0.025)$, but not in the monospecific high-titer samples (Spearman $\mathrm{r}=-0.176 ; \mathrm{p}=$ 0.4 ; data not shown).

These results underscore the findings of unique epitope exposure as defined by the mAbs (Figure 2) and may be indicative of three major immunodominant epitopes relevant to the human anti-MOG response: [1] one epitope exclusively exposed on $\mathrm{rhMOG}_{118}$, [2] one exclusively exposed on $\mathrm{rhMOG}_{125}$, and [3] finally one shared epitope exposed on both $\mathrm{rhMOG}_{118}$ and $\mathrm{rhMOG}_{125}$. None of these seem to be sufficiently exposed on ratMOG 125 . Overall, the anti-MOG reactivity in humans with high-titer antibody responses appears to be diverse and not exclusively directed against epitopes that are commonly exposed (as defined by the $\mathrm{mAb} 8.18 \mathrm{c} 5$ or the Fab M26).

\section{Clinical correlations of anti-MOG IgG}

In neither the entire MS cohort, nor in any of the subtypes (RR-, SP-, PP-MS individually) were anti-MOG antibody titers correlated with the degree of disability as measured by the extended disability status scale (EDSS) (depicted for RR-MS in Figure 4A) when considering the whole cohorts. In contrast, when isolating the subtype of RR-MS samples with high-titer reactivity, their cumulative anti-MOG IgG concentrations, i.e. the sum of the respective high-titer anti-MOG IgG concentrations $\left(\mathrm{rhMOG}_{118}, \mathrm{rhMOG}_{125}\right.$, ratMOG 125 , where applicable), positively correlated with the EDSS at blood sampling (Spearman $r=0.574 ; \mathrm{p}=0.025$; Figure 4B). There was no correlation observed for samples of MS patients with chronic disease. Neither age, gender nor disease duration were identified as confounding factors for the EDSS correlation. 
Table 1 Differential reactivity against the three different MOG isoforms in samples with high-titer reactivity

\begin{tabular}{|c|c|c|c|c|c|c|c|c|c|c|}
\hline & \multirow{2}{*}{$\begin{array}{l}\text { Percentage of samples } \\
\text { tested (proportion) }\end{array}$} & \multicolumn{4}{|c|}{ Monospecific (1 antigen only) } & \multicolumn{4}{|c|}{ Oligospecific (2 antigens) } & \multirow{2}{*}{$\begin{array}{l}\text { All } 3 \text { antigens } \\
\text { rhMOG }_{118} \\
\text { rhMOG }_{125} \\
\text { ratMOG } \\
\%(n)\end{array}$} \\
\hline & & $\%$ & $\begin{array}{l}\mathrm{rhMOG}_{118} \\
(\mathrm{n})\end{array}$ & $\begin{array}{l}\mathrm{rhMOG}_{125} \\
(\mathrm{n})\end{array}$ & $\begin{array}{l}\operatorname{ratMOG} \\
\text { (n) }\end{array}$ & $\%$ & $\begin{array}{c}\text { rhMOG }_{118} \\
+ \\
\text { rhMOG }_{125} \\
\text { (n) }\end{array}$ & $\begin{array}{c}\operatorname{rhMOG}_{118} \\
+ \\
\operatorname{ratMOG}_{125} \\
\text { (n) }\end{array}$ & $\begin{array}{c}\operatorname{rhMOG}_{125} \\
+ \\
\operatorname{ratMOG}_{125} \\
\text { (n) }\end{array}$ & \\
\hline $\mathrm{HC}$ & $10.4(17 / 169)$ & 47.1 & 5 & 1 & 2 & 47.1 & 7 & 1 & 0 & $5.9(1)$ \\
\hline CIS & $7.3(5 / 69)$ & 80.0 & 3 & 1 & 0 & 20.0 & 1 & 0 & 0 & 0 \\
\hline MS & $6.8(22 / 325)$ & 68.2 & 9 & 6 & 0 & 31.8 & 5 & 2 & 0 & 0 \\
\hline $\begin{array}{l}\text { RR- } \\
\text { MS }\end{array}$ & $7.8(15 / 192)$ & 66.7 & 6 & 4 & 0 & 33.3 & 4 & 1 & 0 & 0 \\
\hline $\begin{array}{l}\text { SP/ } \\
\text { PP- } \\
\text { MS }\end{array}$ & $5.3(7 / 133)$ & 71.4 & 3 & 2 & 0 & 28.6 & 1 & 1 & 0 & 0 \\
\hline
\end{tabular}

Percentage of samples with identified high-titer reactivity to have high-titer reactivity exclusively to one MOG isoform, two of the MOG isoforms used or all three MOG isoforms, respectively. There are no significant differences between HC, CIS and MS or any of the MS subgroups ( $p>0.05, \chi^{2}$ test). Samples were deemed high-titer reactive, if the respective $B R$ at the $1 / 800$ serum dilution was above the defined cut-off $B R s\left(5.8,5.6,9.4\right.$ for rhMOG ${ }_{118}$, rhMOG ${ }_{125}$ and ratMOG 125 ).

${ }^{a}$ refer to additional file 2 for characteristics of all samples tested.

For six RR-MS patients longitudinal blood samples were analyzed in a single ELISA assay and demonstrated no significant variation in anti-MOG antibody titers over time (additional file 4). This indicates that pending exceptions, these titers are quite stable over time, excluding titer variability at blood sampling (for example, elevated total IgG due to infection) and that few blood sample measurements would be necessary to define the level of anti-MOG antibody reactivity.

\section{Discussion}

Numerous studies have attempted to assess the presence and clinical implications of serum antibodies against MOG in MS reporting a wide range for the prevalence of anti-MOG antibodies (0\% - 78\%) (reviewed in $[7,8]$ ). The reason for this variability are likely differences in patient populations, assay techniques (e.g., Western blotting, ELISA, RIA), and MOG antigen preparations used $[7,16]$. Furthermore, an important factor to be taken into consideration is the biophysical environment of MOG in the respective assay system. In particular, denaturing conditions may render proteins linearized and thus inaccessible to pathogenic antibodies targeting conformation-dependent epitopes.

The approach presented here addresses two critical criteria: the diversity of the conformational MOG epitope repertoire at the molecular level, and implementation of a fully quantitative assay of the MOG-directed antibody response. In a preliminary experiment we demonstrated that in our ELISA assay antigen coating does not denature MOG (Figure 1), but rather harsh chemical forces would be necessary to fully linearize MOG rendering it inaccessible for antibodies specific for conformational MOG epitopes. We are thus confident that potentially pathogenic conformational anti-MOG antibodies can be detected by our ELISA. We are therefore able to stratify our analyses according to parameters of potential pathological relevance, an essential step towards meaningful interpretation of serum antibodies. We and others have previously shown that single target antigen ELISA may not sufficiently discriminate between pathogenic anti-MOG antibodies, naturally occurring low-affinity autoantibodies or non-pathogenic antibodies directed against linear MOG epitopes $[3,9,17]$. To further emphasize the importance of antibody epitope characterization, we have recently reported in CIS patients a high prevalence of antibodies against native, membrane-embedded MOG that do not fully cross-react with recombinant MOG preparations as demonstrated by cytometry and competition assays [18].

The most relevant finding of this study was derived from $8.2 \%$ of samples that were identified as high-titer reactive. In these samples, we set out to further characterize the diversity of the human serum anti-MOG antibody repertoire with respect to binding to conformational epitopes that was otherwise masked in the entire cohort predominantly consisting of low-titer anti-MOG reactive antibodies (table 1 Figure 3). At least three immunodominant epitopes became apparent, distinctly exposed on three different recombinant MOG "isoforms". These isoforms display only minor differences in terms of amino acid sequence (table 1) and have no other purpose than to rigorously define specific epitopes. These findings of distinct epitope exposure were corroborated using a panel of differentially reactive anti-MOG monoclonal agents (Figure 2) [10]. Importantly, we present data (Figure 1) and have previously shown that relevant conformational MOG epitopes are preserved in ELISA systems [3,10]. Only after isolating high-titer samples, a strong association of circulating anti-MOG IgG with disability in RR-MS patients could be unmasked (Figure 4). This was achieved by 


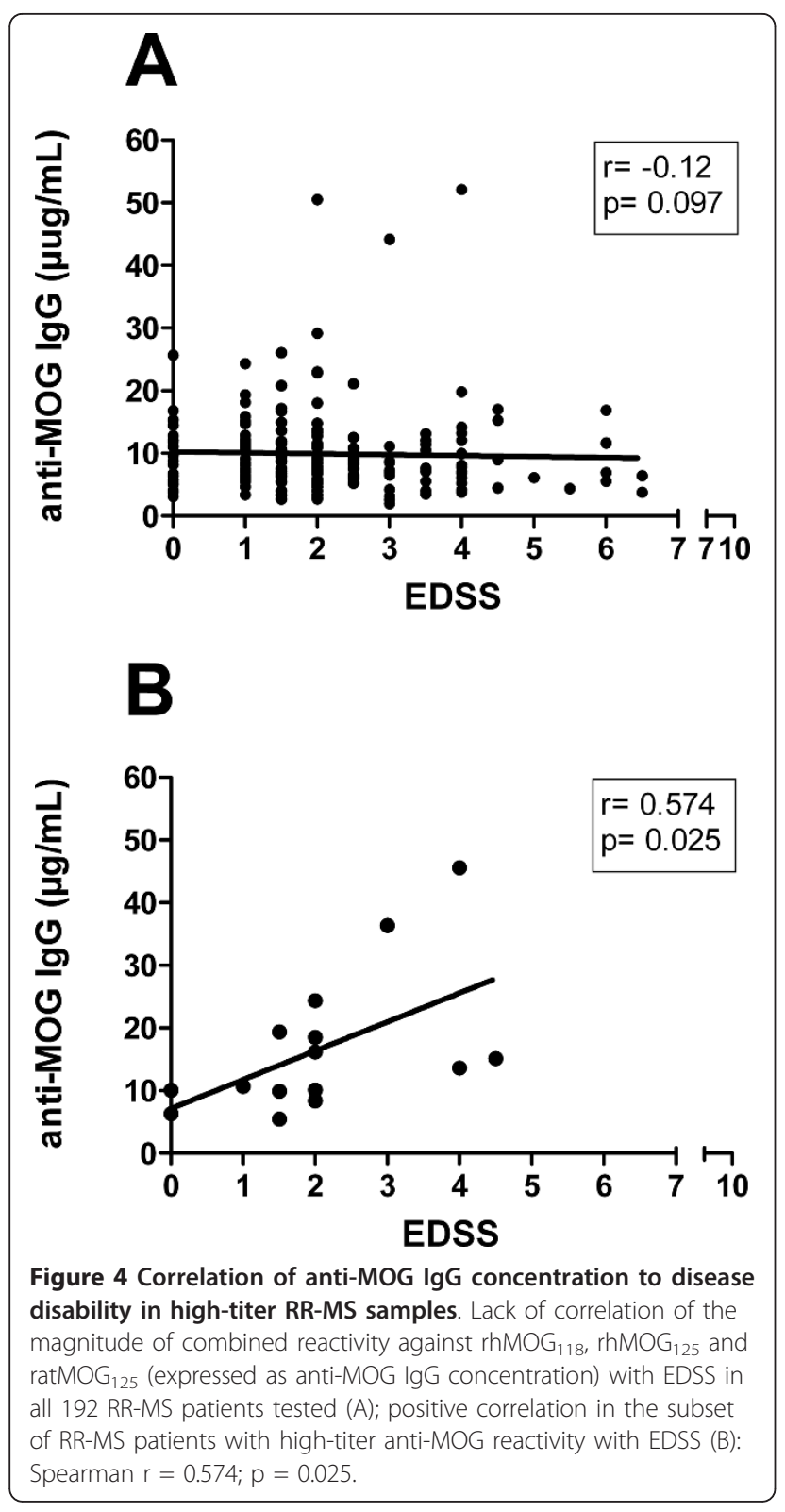

combining the antibody reactivity against all identified putative target epitopes. RatMOG 125 was included in our panel of MOG antigens in order to assess the reactivity against an ubiquitously exposed common epitope, and because of a previously demonstrated cross-reactivity between ratMOG 125 and human anti-MOG IgG [10]. The proportion of high-titer samples appears low at first sight; it is however, quite consistent with recent findings of the frequencies of MOG-reactive $\mathrm{T}$ cells in the peripheral blood of MS patients, that ranged between approximately $2-4$ percent of $T$ cells $[19,20]$. Interestingly and complementing our data, the frequency of these specific $\mathrm{T}$ cells correlated to disease activity and disability [19].
It was an unexpected finding that reactivity to epitopes conserved between species, such as those defined by $8.18 \mathrm{c} 5$ or M26 was not predominant in MS high-titer samples (Figure $2 \mathrm{~A}+\mathrm{B}$, table 1 ). Rather, specific reactivity appeared to be exclusively directed against restricted epitopes of human MOG (table 1). In contrast, humoral responses in rodent EAE are predominantly directed against the 8.18c5 defined epitope [6].

This diversity in epitope recognition may explain why previous studies employing different MOG preparations have generated such divergent results $[7,16]$. Indeed, only one previous study has reported a comparable clinical correlation in 262 patients with MS of which 14\% were deemed anti-MOG positive by comparison to the HC samples [21]. Most other studies have tested considerably smaller numbers of MS patients, and may have not achieved sufficient statistical power to reveal correlations with clinical parameters. To put in perspective, in Type I diabetes mellitus, large sample numbers of 1,300 to over 4,000 per study were needed to identify the prognostic value of autoantibodies in offspring of diabetic patients, with less than $5 \%$ of samples showing high-titer reactivity against the respective antigens [22].

We are aware, that our study does not sufficiently address the issue of specificity that has lacked in previous assays too $[7,8]$, as exemplified by similar overall frequencies of high-titer reactivity in $\mathrm{HC}$ compared to MS and CIS (Figure 3; table 1). However, it is striking, that high-titer anti-MOG reactivity in $\mathrm{HC}$ includes epitopes of rodent MOG, while that in MS and CIS patients does not. While our study was clearly not designed to prove pathogenicity of anti-MOG antibodies as shown previously by passive transfer experiments in animals $[3,23]$, our findings do suggest, that potentially pathogenic anti-MOG antibody responses are highly specific for certain epitopes of human MOG. In this context it was recently shown that demyelination is augmented in an animal model of virally induced mild demyelination if the animals are engineered to produce anti-MOG IgG prior to infection [24]. Thus, it is intriguing to speculate that anti-MOG antibodies in HC render the same pathogenic potential as in MS patients, but $\mathrm{HC}$ individuals have not encountered the pathogenetically relevant viral infection. Furthermore, we cannot rule out the possibility, that the high-titer anti-MOG response occurs secondary to acquired myelin destruction and may as such reflect the magnitude of clinical disability [25].

In summary, we demonstrate for the first time that target epitopes of autoantibody responses are differentially exposed and that the immune response in humans is restricted to distinct epitopes but not identical in all patients and certainly not identical to rodent EAE. Only in a fraction of samples a strong 
and sustained anti-MOG response can be detected, deemed high-titer reactivity. We report that in RR-MS patients with such reactivity the amount of anti-MOG antibodies are correlated with disability. Our findings bear significant clinical relevance that may have been overlooked in previous studies due to smaller sample sizes. This study supports the concept that establishing certain serum autoantibodies as predictive biomarkers in MS may be possible.

\section{Additional material}

Additonal file 1: SDS-PAGE and CD-spectroscopy of MOG. Figures of a SDS-PAGE demonstrating high purity of the three recombinant MOG isoforms, and of a circular dichroism spectroscopy experiment proving correct $\beta$-sheet folding.

Additonal file 2: Clinical characteristics of healthy control, CIS and MS samples tested. Table compiling the demographic and clinical features of the three patient groups.

Additonal file 3: Dilution series of samples with high titers. Figure of an ELISA of two-fold serial serum dilutions against the three MOG isoforms to demonstrate sustained ELISA reactivity of samples with high IgG concentrations ( $>95^{\text {th }}$ percentile) beyond dilutions of $1 / 2,000$.

\section{Additonal file 4: Persistent anti-MOG reactivity in serial samples} Figure of an ELISA reactivity against rhMOG 118 of six samples, for which longitudinal samples were drawn every three months over 18 months; the data prove that anti-MOG IgG concentrations vary only within the limits of the assay.

\begin{abstract}
Abbreviations
ANOVA: analysis of variance; BR: binding ratio; BSA: bovine serum albumin; CD: circular dichroism; CIS: clinically isolated syndrome; CNS: central nervous system; EAE: experimental allergic encephalomyelitis; EDSS: expanded disability status scale; HC: healthy control; $\mathrm{HT}$ : high-titer; mAb: monoclonal antibody; MOG: myelin oligodendrocyte glycoprotein; rMOG: recombinant rat MOG (extracellular domain); MS: multiple sclerosis; OD: optical density; PBS: phosphate buffered saline; PP-MS: primary progressive MS; RR-MS: relapsing remitting MS; SNK: student Newman-Keuls test; SP-MS: secondary progressive MS.
\end{abstract}

\section{Acknowledgements \\ T.M. and P.H.L. were postdoctoral research fellows of the National Multiple Sclerosis Society. \\ We are indebted to Ishita Barman for recombinant MOG preparation and to Drew Dover and Lev Igoudin for performing the assay. Dr. Win Than helped with the denaturing ELISA assay. Lars Lüers oversaw the CD-spectroscopy at the biophysical research core facility in Düsseldorf. \\ We thank the UCSF MS Center staff and neurologists, and Dr. Pablo Villoslada (University of Navarra, Spain) for sample collection and extended clinical examination. \\ This work has been supported by grants from the National Institutes of Health (NS4678-01 to CPG); the National Multiple Sclerosis Society (RG3370- A-3 and 3438-A-7 to CPG); EMD-Serono; the Cure MS Now fund, the Lunardi fund and the Nancy Davis Center Without Walls.}

\section{Author details}

'Neuroimmunology Laboratories, Department of Neurology, University of California San Francisco, CA, USA. ${ }^{2}$ Department of Neurology, Medical Faculty, Heinrich-Heine-University, Düsseldorf, Germany. ${ }^{3}$ Department of Neurosciences, Division of Neurology, Faculty of Medicine, University of Geneva, Switzerland and Department of Pathology and Immunology, Faculty of Medicine, University of Geneva, Switzerland.

\section{Authors' contributions}

TM designed the study, performed the experiments, analyzed data, wrote the paper. PHL analyzed data, wrote the paper and performed experiments. $\mathrm{H}-\mathrm{CVB}$ performed experiments, wrote the paper and reviewed the manuscript. CPG wrote the paper and reviewed the manuscript. TM and CPG had full access to all of the data in the study and take responsibility for the integrity of the data and the accuracy of the data analysis. All authors read and approved the final manuscript.

\section{Competing interests}

The authors declare that they have no competing interests.

Received: 12 August 2011 Accepted: 17 November 2011 Published: 17 November 2011

\section{References}

1. Johns TG, Bernard CC: The structure and function of myelin oligodendrocyte glycoprotein. J Neurochem 1999, 72:1-9.

2. von Büdingen HC, Tanuma N, Villoslada P, Ouallet JC, Hauser SL, Genain CP: Immune responses against the myelin/oligodendrocyte glycoprotein in experimental autoimmune demyelination. J Clin Immunol 2001, 21:155-170.

3. von Büdingen $H C$, Hauser SL, Ouallet JC, Tanuma N, Menge T, Genain CP: Frontline: Epitope recognition on the myelin/oligodendrocyte glycoprotein differentially influences disease phenotype and antibody effector functions in autoimmune demyelination. Eur J Immunol 2004, 34:2072-2083.

4. Mathey E, Breithaupt C, Schubart AS, Linington C: Commentary: Sorting the wheat from the chaff: identifying demyelinating components of the myelin oligodendrocyte glycoprotein (MOG)-specific autoantibody repertoire. Eur J Immunol 2004, 34:2065-2071.

5. Breithaupt C, Schubart A, Zander H, Skerra A, Huber R, Linington C, Jacob U: Structural insights into the antigenicity of myelin oligodendrocyte glycoprotein. Proc Natl Acad Sci USA 2003, 100:9446-9451.

6. Breithaupt C, Schafer B, Pellkofer H, Huber R, Linington C, Jacob U: Demyelinating myelin oligodendrocyte glycoprotein-specific autoantibody response is focused on one dominant conformational epitope region in rodents. J Immunol 2008, 181:1255-1263.

7. Reindl $M$, Khalil $M$, Berger T: Antibodies as biological markers for pathophysiological processes in MS. J Neuroimmunol 2006, 180:50-62.

8. Bielekova B, Martin R: Development of biomarkers in multiple sclerosis. Brain 2004, 127:1463-1478.

9. Menge T, von Büdingen HC, Lalive PH, Genain CP: Relevant antibody subsets against MOG recognize conformational epitopes exclusively exposed in solid-phase ELISA. Eur J Immunol 2007, 37:3229-3239.

10. von Büdingen HC, Hauser SL, Fuhrmann A, Nabavi CB, Lee JI, Genain CP. Molecular characterization of antibody specificities against myelin/ oligodendrocyte glycoprotein in autoimmune demyelination. Proc Natl Acad Sci USA 2002, 99:8207-8212.

11. O'Connor KC, Appel H, Bregoli L, Call ME, Catz I, Chan JA, Moore NH, Warren KG, Wong SJ, Hafler DA, Wucherpfennig KW: Antibodies from inflamed central nervous system tissue recognize myelin oligodendrocyte glycoprotein. J Immunol 2005, 175:1974-1982.

12. Gori F, Mulinacci B, Massai L, Avolio C, Caragnano M, Peroni E, Lori S, Chelli M, Papini AM, Rovero P, Lolli F: IgG and IgM antibodies to the refolded MOG(1-125) extracellular domain in humans. J Neuroimmunol 2011, 233:216-220

13. Linnington C, Webb M, Woodhams PL: A novel myelin-associated glycoprotein defined by a mouse monoclonal antibody. J Neuroimmunol 1984, 6:387-396.

14. Poser CM, Paty DW, Scheinberg L, McDonald WI, Davis FA, Ebers GC, Johnson KP, Sibley WA, Silberberg DH, Tourtellotte WW: New diagnostic criteria for multiple sclerosis: guidelines for research protocols. Ann Neurol 1983, 13:227-231.

15. McDonald WI, Compston A, Edan G, Goodkin D, Hartung HP, Lublin FD, McFarland HF, Paty DW, Polman CH, Reingold SC, Sandberg-Wollheim M, Sibley W, Thompson A, van den Noort S, Weinshenker BY, Wolinsky JS: Recommended diagnostic criteria for multiple sclerosis: guidelines from the International Panel on the diagnosis of multiple sclerosis. Ann Neurol 2001, 50:121-127. 
16. Amor S, Giovannoni G: Antibodies to myelin oligodendrocyte glycoprotein as a biomarker in multiple sclerosis-are we there yet? Mult Scler 2007, 13:1083-1085.

17. Marta CB, Oliver AR, Sweet RA, Pfeiffer SE, Ruddle NH: Pathogenic myelin oligodendrocyte glycoprotein antibodies recognize glycosylated epitopes and perturb oligodendrocyte physiology. Proc Natl Acad Sci USA 2005, 102:13992-13997.

18. Lalive PH, Menge T, Delarasse C, Della GB, Pham-Dinh D, Villoslada P, von Budingen HC, Genain CP: Antibodies to native myelin oligodendrocyte glycoprotein are serologic markers of early inflammation in multiple sclerosis. Proc Natl Acad Sci USA 2006, 103:2280-2285.

19. Bahbouhi B, Pettre S, Berthelot L, Garcia A, Elong NA, Degauque N, Michel L, Wiertlewski S, Lefrere F, Meyniel C, Delcroix C, Brouard S, Laplaud DA, Soulillou JP: T cell recognition of self-antigen presenting cells by protein transfer assay reveals a high frequency of anti-myelin $\mathrm{T}$ cells in multiple sclerosis. Brain 2010, 133:1622-1636.

20. Raddassi K, Kent SC, Yang J, Bourcier K, Bradshaw EM, Seyfert-Margolis V Nepom GT, Kwok WW, Hafler DA: Increased Frequencies of Myelin Oligodendrocyte Glycoprotein/MHC Class II-Binding CD4 Cells in Patients with Multiple Sclerosis. J Immunol 2011, 187:1039-1046.

21. Mantegazza R, Cristaldini P, Bernasconi P, Baggi F, Pedotti R, Piccini I, Mascoli N, Mantia LL, Antozzi C, Simoncini O, Cornelio F, Milanese C: AntiMOG autoantibodies in Italian multiple sclerosis patients: specificity, sensitivity and clinical association. Int Immunol 2004, 16:559-565.

22. Achenbach P, Ziegler AG: Diabetes-related antibodies in euglycemic subjects. Best Pract Res Clin Endocrinol Metab 2005, 19:101-117.

23. Zhou D, Srivastava R, Nessler S, Grummel V, Sommer N, Bruck W, Hartung HP, Stadelmann C, Hemmer B: Identification of a pathogenic antibody response to native myelin oligodendrocyte glycoprotein in multiple sclerosis. Proc Natl Acad Sci USA 2006, 103:19057-19062.

24. Burrer R, Buchmeier MJ, Wolfe T, Ting JP, Feuer R, Iglesias A, von Herrath MG: Exacerbated pathology of viral encephalitis in mice with central nervous system-specific autoantibodies. Am J Pathol 2007, 170:557-566.

25. Antel JP, Bar-Or A: Do myelin-directed antibodies predict multiple sclerosis? N Engl J Med 2003, 349:107-109.

doi:10.1186/1742-2094-8-161

Cite this article as: Menge et al: Conformational epitopes of myelin oligodendrocyte glycoprotein are targets of potentially pathogenic antibody responses in multiple sclerosis. Journal of Neuroinflammation $20118: 161$

\section{Submit your next manuscript to BioMed Central and take full advantage of:}

- Convenient online submission

- Thorough peer review

- No space constraints or color figure charges

- Immediate publication on acceptance

- Inclusion in PubMed, CAS, Scopus and Google Scholar

- Research which is freely available for redistribution

Submit your manuscript at www.biomedcentral.com/submit 